HANNA KRAJEWSKA

(Polska Akademia Nauk, Archiwum w Warszawie)

\title{
MATERIALIEN \\ ZUR GESCHICHTE DER UNIVERSITÄTEN \\ IM ARCHIV \\ DER POLNISCHEN AKADEMIE DER WISSENSCHAFTEN
}

Die schriftlich festgehaltene Geschichte der Universitäten wurde nicht nur in den Universitätsarchiven verwahrt. Es gibt unterschiedliche Gründe, die dazu geführt haben, dass diese Dokumentation zerstreut vorliegt. Hierzu zählen u.a. Kriege, Grenzverschiebungen, teilweises Verlorengehen, Schicksalsfälle und vieles andere mehr. Eine besondere Fundstelle ist meines Erachtens das Archiwum Polskiej Akademii Nauk (das Archiv der Polnischen Akademie der Wissenschaften ${ }^{1}$ ). Dieses Archiv besitzt eine umfangreiche Dokumentation, anhand welcher die Geschichte verschiedener Universitäten - von polnischen wie von ausländischen - erforscht werden kann.

Das Archiv wurde durch den Beschluss Nr. 167/53 des Wissenschaftlichen Sekretariats des PAN-Präsidiums vom 1. Dezem-

1 Künftig: Archiv der PAN. 
ber 1953 gegründet. Sein Sitz befindet sich in Warszawa/Warschau, es hat auch Niederlassungen in Poznań/Posen und Wodzisław Śląski/Loslau (in Oberschlesien). Durch Entscheidungen des Generaldirektors der Staatlichen Archive erlangte das Archiv der PAN den Status einer Stelle mit anvertrauten Beständen und das Recht auf ewiges Verwahren von Dokumenten, die einen Teil der staatlichen Archivbestände darstellen. Innerhalb der PAN hat es den Status einer wissenschaftlichen Stelle mit Unterstützungscharakter.

Satzungsgemäß sammelt das Archiv die Akten der PAN, der Kanzlei, Institute und anderer Stellen, wie z.B. des Botanischen Gartens, der Bibliothek von Kórnik sowie des Muzeum Ziemi (Naturhistorisches Museums). Einen wichtigen Teil der Sammlungen stellt die Dokumentation der Gesellschaften und sonstiger wissenschaftlicher Einrichtungen, darunter auch ein gewisser Teil der Universitätsarchivalien dar. Wesentliche Bedeutung für die Sammlungen als Ganzes haben die Nachlässe der polnischen Gelehrten, von denen das Archiv der PAN bereits mehr als 500 besitzt. All dieses Material gelangte hierher durch Erwerb, Depot oder Schenkungen. Gezielte Erwerbungen überwogen in den 1990er Jahren, heute sind es vor allem Schenkungen.

Im Archiv befindet sich auch eine Sammlung von etwa 30000 Fotographien, zumeist mit Bezug auf wissenschaftliche Ereignisse, zu Wissenschaftsgebäuden oder zu einzelnen Wissenschaftspersönlichkeiten. Es gibt auch eine Sammlung von Medaillen, die zu Ehren eines Gelehrten, der wissenschaftlichen Einrichtungen oder im Zusammenhang mit Jubiläen von wissenschaftlichen Ereignissen, Kongressen oder Tagungen geprägt worden waren.

In den Sammlungen des Archivs der PAN werden Informationen in Bezug auf 79 polnische und ausländische Universitäten, 10 Technische Universitäten und 32 Hochschulen verwahrt. Der Wert dieser Dokumentation und auch die Anzahl der Aktenein- 
heiten sind unterschiedlich. Größtenteils ist es das Material mit Informationen über einen größeren oder kleineren Teilbereich der Tätigkeit der jeweiligen Universität. Zum überwiegenden Teil handelt es sich um Originale. Es handelt sich ohne Frage um eine einzigartige Sammlung, die eine umfangreiche Quellenbasis für Forschungen insbesondere zur Geschichte der Wissenschaft in Polen und der dortigen Universitäten ist.

Eine sehr interessante Dokumentensammlung betrifft die Johann-Kasimir-Universität Lemberg, eine der ältesten in Osteuropa gegründeten Universität und die vierte auf dem Gebiet der ehemaligen polnischen Republik. Gegründet wurde sie 1661 von König Johann Kasimir. Um die Wende des 19. zum 20. Jahrhundert wurde diese Universität, damals innerhalb der Grenzen des österreichischen Teilungsgebiets, zur größten Universität in Galizien und zweitgrößten in Österreich. Nach der Wiedererlangung der Unabhängigkeit wurde dieser Universität der Namen ihres Gründers - König Johann Kasimir - durch Beschluss der polnischen Regierung verliehen. An dieser Universität hielten hervorragende Wissenschaftler mit weltweitem Ruf Vorlesungen. Es entstanden starke Lemberger Wissenschaftlermilieus, die aktiv neue Zentren - Schulen genannt - gründeten: Lemberger mathematische Schule, Lemberg-Warschauer philosophische Schule, Lemberger anthropologische, geographische, zoologische Schule. Nach dem Zweiten Weltkrieg hat man auf der Grundlage dieser alten Universität die ukrainische Iwan-Franko-Universität gegründet. Im Archiv der PAN, in der Gruppe der kleinen polnischen Verbände von wissenschaftlichen Einrichtungen und Organisationen in Lemberg, befindet sich die Dokumentation, die die Johann-Kasimir-Universität in Lemberg aus den Jahren zwischen den beiden Weltkriegen betrifft: Akademische Akten des historischen und naturwissenschaftlichen Zirkels, Fragmente des allgemeinen Schriftverkehrs, Unterlagen einiger Universitätsein- 
richtungen, u.a.: des Instituts für Neuzeitgeschichte, Instituts für Orientalistik, Referate der Professoren, Anordnungen, Rundschriften, Bekanntmachungen des Ministeriums für Religionsbekenntnisse und öffentliche Aufklärung sowie der Universitätsorgane, z.B. in Sachen der Reform des Magisterprüfungssystems. Schon diese kurze Aufstellung zeigt, dass diese Unterlagen in Original und in Kopie vorliegen. Die Kopien entstammen den Unterlagensammlungen des Ministeriums. Bedenkt man jedoch, dass infolge der Vernichtung im Zweiten Weltkrieg nur wenige Ministerialakten erhalten blieben, können die Dokumente als eine Vervollständigung angesehen werden, ja fallweise sogar als einzig erhaltene Exemplare dienen.

Die Informationen zum Thema der Universitätstätigkeit sind auch in vielen Nachlässen der mit Lemberg verbundenen Gelehrten, die an dieser Universität tätig waren und Vorträge hielten, zu finden.

Die Nachlässe sind eine Quellenschatzgrube. Es gibt hier viele Informationen zum Autor selbst, zu seiner wissenschaftlichen Tätigkeit, seinen Interessen. Oft findet die Berufstätigkeit an einer Universität, insbesondere bei der Ausübung der Funktion eines Rektors, Dekans oder Institutsdirektors, einen starken Widerhall in dem privaten Nachlass der Wissenschaftler. Diese Verflechtung erschwert oftmals die Unterscheidung zwischen den strikt privaten und den Dienstunterlagen. Dies ist besonders im Schriftverkehr auffallend.

Mit der Lemberger Universität waren viele hervorragende Persönlichkeiten, deren persönliche Papiere und deren Schriftverkehr sich im Archiv der PAN befinden, verbunden. $\mathrm{Zu}$ ihnen zählen Henryk Arctowski, ein Geograph, Geologe, Reisender, Forscher der Polarländer, Stefan Banach, der hervorragende polnische Mathematiker, Benedykt Dybowski, ein Zoologe, der die Fauna von Baikal-See und Kamtschatka erforschte. Im Nach- 
lass des letztgenannten gibt es u.a.: Xerokopien der Akten zur politischen Tätigkeit während seines Studiums an der Dorpater Universität. Die Kopien entstammen der Sammlung des Historischen Archivs von Estland in Dorpat.

Im Archiv der PAN gibt es auch viele Abschriften von den nicht mehr existierenden, im Zweiten Weltkrieg vernichteten Akten. Welch bedeutende Rolle die Kopien bei der Vergangenheitsaufarbeitung spielen, brauche ich hier wohl nicht auszuführen.

Ein weiterer Nachlass von Wiktor Hahn, einem Literaturhistoriker, Bibliographen, Kenner der Werke unseres Dichters Juliusz Słowacki, enthält Materialien der von ihm organisierten Jubiläumsfeier zu Ehren dieses Dichters in Lemberg. Erhalten blieb auch eine, mit 3500 Briefen sehr umfangreiche Korrespondenz mit hervorragenden polnischen Schriftstellern und Gelehrten.

Im Nachlass von Mieczysław Kreutz, einem Psychologen und Pädagogen, befinden sich die Akten und die Chronik der Anstalt für Psychologie an der Universität Lemberg. Zwischen den persönlichen Papieren Karol Koranyis, eines Juristen, Strafrechtshistorikers und Rektors der Lemberger Universität, sind Unterlagen zu seiner Tätigkeit an der Universität, hierunter Zeugnisse und Diplome in Original, zu finden. Der Professor war während des Krieges an der Iwan-Franko-Universität tätig und wechselte danach an die Nikolaus-Kopernikus-Universität Toruń/Thorn. Dokumente des ähnlichen Typs enthält der Nachlass von Hugo Steinhaus, einem hervorragenden Mathematiker, der nach dem Kriege an die Universität Wrocław wechselte.

Kazimierz Twardowski, ein berühmter Philosoph, der Begründer der Lemberg-Warschauer philosophischen Schule, auch ein Rektor, hinterließ Unterlagen zur organisatorischen und didaktischen Tätigkeit an der Universität, darunter u.a. politische Bewertungen mancher Professoren. Den interessantesten Teil stellen 
die Tagebücher des Professors aus der Jugendzeit, des Ersten Weltkrieges und der Zeit danach bis zum Zweiten Weltkrieg dar. Für Forscher sind sie eine nicht zu unterschätzende Informationsquelle. Ähnliche Bedeutung haben vorhandene Fragmente des Tagebuchs von Professor Józef Kallenbach, die die Universitäten in Vilnius und Krakau sowie die polnische Staatsbildung nach dem Ersten Weltkrieg behandeln.

Die Universität Vilnius ist die nächste Universität, die eine umfangreiche Dokumentation in den Sammlungen des Archivs der PAN hat. Diese Universität wurde 1579 durch den König Stephan Báthory gegründet und in demselben Jahr von Papst Gregor XIII. offiziell bestätigt. Die größte Blütezeit erreichte die Universität im 17. Jahrhundert; während der Teilungen Polens war sie eine kurze Zeit lang tätig, um dann 1919 wiederhergestellt zu werden. Dann nannte man sie Stephan-Báthory-Universität. Nach dem Zweiten Weltkrieg kam der überwiegende Teil der überlebenden Wissenschaftler, des Verwaltungspersonals und der Studenten, aus ihrem Heimatsgebiet zwangsweise ausgesiedelt, nach Thorn, wo die Nikolaus-Kopernikus-Universität gegründet wurde und wohin auch die Überreste aus der Universität Lemberg zum Teil gelangten.

Im Archiv der PAN befindet sich eine Kollektion der Dokumente zur Geschichte der Universität Vilnius. Es handelt sich um eine Sammlung von Schreiben, von Korrespondenzen, von Plänen, Zeichnungen, Abschriften und Fotographien. Bemerkenswert ist das Originaldokument betreffend die Gebäude der Akademie Vilna aus dem Jahre 1580, unterzeichnet von Stephan Báthory, ferner das Schreiben des Rektors Jan Śniadecki aus dem Jahre 1808, oder das Schreiben des Kurators Adam J. Czartoryski über die Aufnahme der Studenten aus dem Gebiet des Herzogtums Warschau von 1810. Aus der Zeit des Wiederaufbaus nach dem Ersten Weltkrieg gibt es noch die Schreiben von Józef 
Piłsudski, darunter ein Brief von 1924 an den Rektor, in dem der Marschall sein Monatsgehalt der Universität spendet. In der Kollektion werden Urkunden betreffend die Gründung der Hochschule nach dem Ersten Weltkrieg und den Vermögensbestand der Universität, Anträge, Ernennungen, Protokolle der Sitzungen vom Senat und Rektorenkonferenz in Xerokopien verwahrt. Die Originale befinden sich im Zentralen Litauischen Staatsarchiv in Vilnius. Die historischen Schicksalswendungen von Vilnius wurden ausschlaggebend für die Zerstreuung der an diesem Standort verfassten und gesammelten Archivalien. Das Schicksal der Stadt teilten auch ihre Einwohner, unter diesen auch die Wissenschaftler, die persönliche Andenken wie auch jene an die Universität, mit der sie verbunden waren, sammelten. Sowohl die Schicksale der Menschen als auch die Schicksale der von ihnen gesammelten Bruchstücke der Vergangenheit fanden ihren Überlebensort im Archiv der PAN. Darunter sind Dokumente von Władysław Dziewulski, dem Rektor und Promotor des Ausbaus des Observatoriums der Astronomischen Universität. In seinem Nachlass sind Berichte, Finanzakten, technische Dokumentationen, Korrespondenzen und Bilder verwahrt. Mehr Informationen zur Universität aus verschiedenen Zeiten ihrer Tätigkeit sind in den Urkunden von Stefan Ehrenkreutz, auch einem Rektor (Korrespondenz über die Übernahme der Universität von den litauischen Behörden 1939), Henryk Elzenberg, dem Philosophen (Tagebücher), Ludwik Chmaj, dem Philosophen (Abschriften und Originale aus den Jahren 1771-1832), Ludwik Kolankowski, dem ersten Rektor der Universität Thorn (Akten der Organisation der Universität Vilnius, Korrespondenz von Marian Morelowski, dem Kunsthistoriker (Vorlesungsskripte), Stefan Rygla, dem Bibliothekar (Tagebücher), oder Marian Zdziechowski, ebenfalls Rektor der Hochschule (Erinnerungen, Korrespondenz) zu finden. 
Die Nachlässe der Rektoren enthalten immer neben dem biographischen Material und Urkunden zur wissenschaftlichen Tätigkeit auch Dokumente, die in Verbindung mit der von ihnen ausgeübten Funktion stehen. Im Archiv der PAN werden Nachlässe von zahlreichen Rektoren der Universitäten Lemberg, Vilnius, Warschau, Posen, Thorn und Jagellonen-Universität in Krakau (15 Nachlässe) verwahrt. In vielen Nachlässen gibt es Informationen über die Universitäten Łódź, Wrocław/Breslau, Technische Universität Gdańsk/Danzig, Warschau u.a.m.

In den privaten Papieren der mit verschiedenen Universitäten verbundenen Gelehrten befinden sich viele Dokumente zu ihrer wissenschaftlich-organisatorischen Tätigkeit. Dazu gehören statistische Materialien, Referate, Vorlesungen, Rezensionen und Gutachten zu den wissenschaftlichen Arbeiten sowie Korrespondenzen. Vorhanden ist hier auch das amtliche Material über die Beschäftigung in verschiedenen Instituten und Anstalten, z.B. die Anfänge der Anstalt für Limnologie der SGGW/Agraruniversität (Marian Gieysztor), Institut für Archäologie Polens der Universität Warschau (Władysław Antoniewicz), Lehrstuhl für Geodätische Astronomie der Technischen Universität Warschau (Felicjan Kępiński), Fakultät für Chemie der Universität Warschau (Władysław Kołos), Historisches Institut der Universität Warschau (Tadeusz Manteuffel). Oftmals enthält diese Dokumentation Protokolle über die Sitzungen des Senats oder einzelner Institute, Informationen über Studienreisen ins Ausland, Berichte über die Rückforderung von Sammlungen, Mitgliedszeugnisse wissenschaftlicher Gremien an verschiedenen ausländischen Universitäten. Das Echo zahlreicher Ereignisse an den Universitäten enthält die Dokumentation der organisatorischen Tätigkeit im Hochschulwesen aus den 30er Jahren des 20. Jahrhunderts, die Akten der Zusammenarbeit mit Entscheidungsträgern zur Entwicklung des Hochschulwesens in den 50er Jahren, ferner die 
Urkunden des Expertenteams für die Wirtschaftsreform oder die Akten der Kommission für Standardisierung der Geographischen Namen.

Außerordentlich interessant sind die Akten zum geheimen Schulwesen und Organisation des Unterrichts während des Zweiten Weltkrieges. In den Nachlässen sind Erinnerungen, Korrespondenzen (Roman Pollak), Prüfungsergebnisse, Protokolle der Seminare (Jakub Sawicki, Rechtshistoriker) erhalten. Bemerkenswert sind die deutschen Akten, hinterlassen von Professor Wolfgang Gleissberg über die Organisation der Reichsuniversität Posen, die sich im Nachlass von Bronisław Niklewski, dem Biologen befinden.

Den Nachlässen ist der Aspekt von Internationalität von Wissenschaftlern zu entnehmen. Sie sprechen Fremdsprachen, reisen durch die Welt, haben immer was Interessantes zu erzählen, besitzen zahlreiche Kontakte. Im Archiv der PAN gibt es auch Nachlässe der Professoren, die ihre Vorlesungen in exotischen Stätten hielten, wie die Universität Kabul (Edward Stenz, Meteorologe, 1938-1958) oder St.-Markus-Universität in Lima (Władysław Folkierski, Mathematiker, Ingenieur, 1841-1904; Ende des 19. Jahrhunderts hielt er Vorträge in Lima und nahm am Eisenbahnbau teil).

Die Vorlesungen wurden jedoch vor allem an vielen europäischen und amerikanischen Universitäten gehalten. Aufgrund der Thematik der Fachtagung lenke ich nun die Aufmerksamkeit auf die Kontakte mit den deutschen Universitäten. Diese Dokumentation besteht u.a. aus Notizen, Bescheinigungen, Diplomen darunter Diplome honoris causa - und Vorlesungen. Sie betrifft die Humboldt-Universität zu Berlin (Emil Chroboczek, Gemüseanbau), die Volkshochschule für die polnischen Aussiedler in Mattenberg bei Kassel (Romuald Gumiński, Klimatologe, 1945-1946). 
Darüber hinaus sind hier die Universitäten in Berlin, Jena, Göttingen, Leipzig mit den Urkunden aus dem Ende des 19. Jahrhunderts und aus der Zeit des Ersten Weltkrieges vertreten, ferner gibt es Materialien zu den Universitäten Heidelberg (honoris causa für Jerzy Pniewski, Superkern-Physiker), Regensburg sowie die deutsche Reichsuniversität Posen (Korrespondenz der Zoologischen Anstalten 1940-1944, Institut für Systematik und Tierevolution in Krakau). Erhalten blieben auch die Vorlesungen von Prof. Henryk Grossman, dem Ökonom, der an den Universitäten Frankfurt am Main und Leipzig in der Zeit zwischen den beiden Weltkriegen tätig war.

Überlieferungen ähnlichen Typs sind aus den Universitäten des gesamten deutschsprachigen Gebiets, etwa aus Österreich, vor allem Wien, und aus der Schweiz - Zürich, Fribourg, Bern - erhalten geblieben. Dieser Teil der Dokumentation informiert überwiegend über umfangreiche internationale Kontakte der polnischen Wissenschaftler. Manchmal findet man hier Material zu besonderen Aktivitäten, z.B. im Nachlass von Wiktor Schramm, dem Ökonom: Hier werden die Schriftstücke zu seiner Tätigkeit in der Internationalen Expertenkommission beim Deutsch-Polnischen Schiedsgericht in Paris, einberufen nach dem Versailler Vertrag zur Abschaffung und Abschätzung der deutschen Vermögen, die sich auf dem polnischen Gebiet nach dem Ersten Weltkrieg befanden, verwahrt. Es handelt sich dabei um die Texte der Gutachten, Protokolle der Kommissionssitzungen, Polemiken, Repliken, Berichte. Vergleichbares Material über die Abschätzung des Fürstentums Krotoschin-Adelnau (poln. Księstwo KrotoszyńskoOdolanowskie) in den gegen den polnischen Staat durch den Prinzen Thurn von Taxis (1925-1929) erhobenen Forderungen findet sich ebenfalls. Dieser Typ von Dokumenten behandelt mehr die polnisch-deutschen Beziehungen als die Dokumentation der 
Hochschule. Dies ist jedoch ein Beispiel für die Verschiedenartigkeit des Engagements polnischer Gelehrten.

Abschließend möchte ich noch einen Typ der Dokumentation erwähnen, den ich bereits genannt habe, ich möchte diesen nun aber in seiner Bedeutung hervorheben. Es ist die umfangreiche Korrespondenz, die sich in jedem Nachlass befindet. Im Archiv der PAN befinden sich nämlich Briefe der Gelehrten aus der ganzen Welt. Unter den wichtigen Namen sind Albert Einstein, Maria Skłodowska-Curie, ihre Tochter Irena und Frédéric JoliotCurie, Paul Langvein, Ernest Rutherford und viele andere mehr. Die Briefe zeigen die Gelehrten in einem anderen Licht als die behördlichen Akten. Sie sind ein umfangreiches Quellenmaterial zu vielen Aspekten des privaten Bereichs und der wissenschaftlichen Tätigkeit.

Die Mehrheit der Dokumentation des Archivs der PAN hat dadurch, dass hier hauptsächlich die Nachlässe der Wissenschaftler gesammelt werden, eher persönlichen Charakter. Die vorhandenen Sammlungen dokumentieren das Leben der Hochschulen, der Universitäten in vielen Detailaspekten und können somit entweder zur Grundlage oder Hilfe zur Vervollständigung für Forscher, die die Geschichte der Universitäten erhellen wollen, genutzt werden. 


\section{MATERIAŁY DO HISTORII UNIWERSYTETÓW \\ W ARCHIWUM \\ POLSKIEJ AKADEMII NAUK}

(STRESZCZENIE)

Materiały do dziejów uniwersytetów zachowały się nie tylko w archiwach uczelnianych. Tak na przykład w Archiwum Polskiej Akademii Nauk znajduje się ogromna dokumentacja dotycząca dziejów różnych uniwersytetów, zarówno polskich, jak i zagranicznych.

Archiwum PAN powstało na podstawie uchwały nr 167/53. Sekretariatu Naukowego prezydium PAN z dnia 1 grudnia 1953 roku. W jego zbiorach przechowywane są informacje dotyczące 79 uniwersytetów, 10 politechnik i 32 innych wyższych uczelni. Wartość tej dokumentacji, a także liczba jednostek aktowych jest różna. W większości są to materiały informujące o jakimś mniejszym lub większym wycinku działalności danej uczelni. W większości są to oryginały. Ten unikatowy zbiór tworzy szeroką bazę źródłową do badań nad dziejami nauki w Polsce, a uniwersytetami w szczególności.

Wśród przechowywanych w Archiwum PAN materiałów wyróżniają się dokumenty dotyczące uniwersytetów Jana Kazimierza we Lwowie, Stefana Batorego w Wilnie, Mikołaja Kopernika w Toruniu i wielu innych. Znaczącą wartość posiadają zwłaszcza spuścizny rektorów uniwersytetów: lwowskiego, wileńskiego, warszawskiego, poznańskiego, toruńskiego i Jagiellońskiego (15 spuścizn). W wielu spuściznach są informacje o uniwersytetach Łódzkim, Wrocławskim, Politechnice Gdańskiej, Śląskiej, Warszawskiej i Wrocławskiej, wyższych szkołach ekonomicznych, handlowych, górniczych, pedagogicznych i rolniczych. Uniwersytety w Berlinie, Jenie, Getyndze, Lipsku reprezentowane są przez materiały z końca XIX wieku oraz z czasów I wojny światowej. Niezmiernie interesujące są akta związane $z$ tajnym nauczaniem i organizacją nauki w czasie II wojny światowej. 
Na wyróżnienie zasługuje ponadto bogata korespondencja przechowywana w Archiwum PAN. Znajdują się tu listy uczonych z całego świata, m.in. Alberta Einsteina, Marii Skłodowskiej-Curie, jej córki Ireny, Fryderyka Joliot-Curie, Paula Langveina czy Ernsta Rutherforda. Listy ukazują tych uczonych w innym świetle niż akta urzędowe. Są bogatym materiałem źródłowym na temat wielu aspektów ich życia prywatnego i działalności naukowej. Większość dokumentacji Archiwum PAN ma charakter osobisty, co jest wynikiem faktu, że gromadzone są tu głównie spuścizny uczonych.

Opracowane przez Autorkę/Prepared by the Author 